\title{
Studies of antimicrobial resistance in rare mycobacteria from a nosocomial environment
}

Sónia Gonçalves Pereira ${ }^{1 \dagger}$, Susana Alarico ${ }^{1,2 \dagger}$, Igor Tiago ${ }^{3}$, Diogo Reis ${ }^{1}$, Daniela Nunes-Costa ${ }^{1,4}$, Olga Cardoso ${ }^{5,6}$, Ana Maranha $a^{1,2^{*}}$ and Nuno Empadinhas ${ }^{1,2^{*}}$ (i)

\begin{abstract}
Background: Nontuberculous mycobacteria (NTM) are ubiquitous in nature and recognized agents of opportunistic infection, which is often aggravated by their intrinsic resistance to antimicrobials, poorly defined therapeutic strategies and by the lack of new drugs. However, evaluation of their prevalence in anthropogenic environments and the associated antimicrobial resistance profiles have been neglected. In this work, we sought to determine minimal inhibitory concentrations of 25 antimicrobials against 5 NTM isolates recovered from a tertiary-care hospital surfaces. Antimicrobial susceptibilities of 5 other Corynebacterineae isolated from the same hospital were also determined for their potential clinical relevance.
\end{abstract}

Results: Our phylogenetic study with each of the NTM isolates confirm they belong to Mycobacterium obuense, Mycobacterium mucogenicum and Mycobacterium paragordonae species, the latter initially misidentified as strains of $M$. gordonae, a species frequently isolated from patients with NTM disease in Portugal. In contrast to other strains, the $M$. obuense and M. mucogenicum examined here were resistant to several of the CLSI-recommended drugs, suggestive of multidrug-resistant profiles. Surprisingly, M. obuense was susceptible to vancomycin. Their genomes were sequenced allowing detection of gene erm (erythromycin resistance methylase) in M. obuense, explaining its resistance to clarithromycin. Remarkably, and unlike other strains of the genus, the Corynebacterium isolates were highly resistant to penicillin, ciprofloxacin and linezolid.

Conclusions: This study highlights the importance of implementing effective measures to screen, accurately identify and control viable NTM and closely related bacteria in hospital settings. Our report on the occurrence of rare NTM species with antibiotic susceptibility profiles that are distinct from those of the corresponding Type strains, along with unexpected resistance mechanisms detected seem to suggest that resistance may be more common than previously thought and also a potential threat to frail and otherwise vulnerable inpatients.

Keywords: Nontuberculous mycobacteria (NTM), Mycobacterium mucogenicum, Mycobacterium obuense, Mycobacterium paragordonae, Conynebacterineae, Antimicrobial resistance

\footnotetext{
*Correspondence: nuno.empadinhas@uc.pt; anamaranha@gmail.com

†Sónia Gonçalves Pereira and Susana Alarico contributed equally to this work

and share first authorship.

${ }^{1}$ Center for Neuroscience and Cell Biology (CNC), University of Coimbra,

3004-504 Coimbra, Portugal

Full list of author information is available at the end of the article
}

(c) The Author(s). 2019 Open Access This article is distributed under the terms of the Creative Commons Attribution 4.0 International License (http://creativecommons.org/licenses/by/4.0/), which permits unrestricted use, distribution, and reproduction in any medium, provided you give appropriate credit to the original author(s) and the source, provide a link to the Creative Commons license, and indicate if changes were made. The Creative Commons Public Domain Dedication waiver (http://creativecommons.org/publicdomain/zero/1.0/) applies to the data made available in this article, unless otherwise stated. 


\section{Background}

Hospitals are major sources of infectious agents with 7 to $10 \%$ of all inpatients estimated to develop at least one hospital associated infection (HAI) during their admission [1-3]. In addition to the debilitated health conditions rendering patients more susceptible to infections, hospital environments represent added risks inflicted by antibiotic resistant opportunistic pathogens $[3,4]$. The World Health Organization (WHO) recently issued its first ever list of antibioticresistant 'priority pathogens', the most prevalent and antibiotic resistant bacterial pathogens associated with nosocomial infections [5]. WHO emphasized "Mycobacteria was not subjected to review for inclusion in this prioritization exercise as it is already a globally established priority for which innovative new treatments are urgently needed" [5]. Most Mycobacterium species are environmental saprophytes designated nontuberculous mycobacteria (NTM) to be distinguished from those that cause tuberculosis [6]. Their ability to infect humans and to colonize man-made environments including hospitals with inadequate disinfection of water distribution systems, as well as an overall resistance to antibiotics and high prevalence of risk factors in the population namely chronic diseases and advanced age, all merge into a potential and serious health threat [7]. The growing number of NTM infections in the lung, soft tissue or skin of susceptible individuals, combined with their known association with nosocomial infections and outbreaks, has brought up a general awareness towards this bacterial group [7]. While clinical laboratories routinely screen for the presence of some important pathogens others are often neglected. These include fastidious and slowly growing pathogens or opportunists. For example, the clinical significance of NTM isolation besides the most prominent pathogens of the group (such as MAC or $M$. abscessus) is not totally understood, especially in lung disease. Most NTM are non pathogenic for healthy people, but almost all can be responsible for opportunistic infections in susceptible individuals. In cases of isolation of NTM usually considering normal colonizers or contaminants, patients require careful clinical evaluation, taking into account factors such as the patient's immunologic status and the site of infection to determine the significance of the isolate $[8,9]$. Potential pathogens can colonize hospital settings but the awareness of their presence is low because they are not routinely screened. In fact, there exists a bias in screening since only about $2 \%$ of microorganisms grow well in standard clinical culture media and are considered significant [10]. Some of the undetected pathogens emerge as cause of atypical infections frequently associated with relevant drug resistance, highlighting the necessity for urgent measures for their routine detection and control. For example, contamination of ICU inanimate surfaces and equipment has been identified as a contributing source of transmission of pathogens to ICU patients in outbreaks [11].
Members of the genus Mycobacterium, which in December 2018 comprised 198 valid species [12], are among a restricted gallery of the most resilient organisms we know of as they can withstand multiple stress conditions such as high temperature, oxidative stress, nutrient deprivation and prolonged desiccation [6]. Other related bacteria from the suborder Corynebacterineae, namely Corynebacterium spp., Nocardia spp., Rhodococcus spp. and Gordonia spp. can also be the cause of atypical infections in susceptible individuals but their routine identification has been neglected in the clinical practice as well $[13,14]$.

The aim of this study was to address the extent of antimicrobial resistance in strains of NTM and other Corynebacterineae isolated from a nosocomial environment. Although health authorities neglect the fact that the prevalence of NTM infection is seriously underestimated in the European Union in general [15] and in Portugal in particular [16], only a strong commitment to NTM research will allow proportional responses to this health threat.

\section{Results}

Distribution of NTM and other actinobacterial isolates, identification and phylogenetic studies

Samples were collected from different sites of 4 hospital wards in 3 sampling events as previously described [17]. All isolates were recovered after 2 or 4 weeks of incubation in Middlebrook 7H10-PANTA medium and none after 6 weeks incubation. Of the actinobacterial isolates 10 belonged to the suborder Corynebacterineae, their correct phylogenetic identification and antibiotic susceptibility pattern were the focus of the present study, to address the extent of antimicrobial resistance in Corynebacterineae isolated from a nosocomial environment. The other 24 isolates belonging to genera Dermacoccus, Kocuria, Microbacterium and Micrococcus (all non-Corynebacterineae) were not identified to the species level and their antibiotic susceptibilities were not examined in this study.

As inferred from $16 \mathrm{~S}$ rRNA phylogenetic analyses, 3 isolates of the 10 Corynebacterineae were related to species Corynebacterium jeikeium, C. amycolatum and $C$. imitans and other 2 isolates were closely related to species Gordonia otitidis and G. sputi (Table 1, Fig. 1).

Five of the Corynebacterineae were classified as members of the genus Mycobacterium, as determined from a phylogenetic tree of concatenated 16S rRNA, hsp65 and $r p o B$ genes (Fig. 2). One isolate was closely related to the species $M$. mucogenicum, other to $M$. obuense and 3 isolates were closely affiliated to the slowly growing species $M$. paragordonae, all nontuberculous mycobacteria (NTM) (Table 1, Fig. 2). Since M. avium is a commonly isolated NTM but was not detected in this study, control growth experiments to assess possible inhibition of growth 
Table 1 NTM and other Corynebacterineae members isolated from different hospital sites (adapted from [16]). Phylogenetic trees in the present study confirm that isolates 10Alll, 29AllI and 35Alll are probably M. paragordonae

\begin{tabular}{llll}
\hline Isolate & Closely related species & Ward & Amenity \\
\hline 1All & Gordonia otitidis & Hematology & Restroom light switch \\
6FIII & Corynebacterium jeikeium & Hematology & Bed table \\
10All & Mycobacterium paragordonae & Hematology & Therapy room bench \\
22DIII & Mycobacterium obuense & Urology & Restroom sink \\
24All & Mycobacterium mucogenicum & Urology & Restroom light switch \\
29AIII & Mycobacterium paragordonae & Renal Transplant Unit & Therapy room bench \\
35All & Mycobacterium paragordonae & Renal Transplant Unit & Bed \\
52Alll & Corynebacterium amycolatum & Medicine A & Bed table \\
55All & Gordonia sputi & Medicine A & Bed hand support \\
58FIII & Corynebacterium imitans & Medicine A & Bed table light switch \\
\hline
\end{tabular}

by Middlebrook 7H10-PANTA were performed with some clinical $M$. avium isolates available in our collection, which ruled out such possibility (results not shown). Corynebacterium and Gordonia isolates were recovered from the Hematology and Medicine A wards (Table 1). NTM isolates were recovered from Hematology $(M$. paragordonae, 10AIII), Urology (M. obuense, 22DIII and M. mucogenicum, 24AIII), and Renal Transplant Unit (M. paragordonae, 29AIII and 35AIII) wards as previously described [17]. All but the $M$. obuense isolate were collected from dry surfaces/equipment.

\section{Determination of minimal inhibitory concentrations (MIC) of antimicrobials}

MIC values of the 25 antimicrobials tested are indicated in Table 2. Considering the Clinical \& Laboratory Standards Institute (CLSI) susceptibility interpretation for the antimicrobials included in the standards, the $M$. obuense isolate exhibited higher resistance levels and was the single isolate resistant to clarithromycin, which could be explained by the presence of the gene erm (erythromycin resistance methylase, accession number MG770427) in the draft genome sequenced in this study. On the other hand, $\operatorname{tet}(V)$ genes associated to tetracycline resistance were detected in the M. obuense and M. mucogenicum isolates (accession numbers MG770425 and MG770428, respectively) but only the latter was resistant to this drug (Table 2). Mycobacterium paragordonae isolates $(n=3)$ were susceptible to amikacin, ciprofloxacin, clarithromycin and linezolid, four of the antimicrobials recommended by CLSI to test drug susceptibility of slowly growing mycobacteria [18]. MICs for the other antimicrobials tested were not possible to interpret. All Corynebacterium isolates were resistant to ciprofloxacin, linezolid, penicillin and to imipenem, although the latter is recommended for Corynebacterium susceptibility testing despite the fact that no interpretative criteria are available (Table 2). Both Gordonia isolates were resistant to imipenem while only one of them (55AIII) was resistant to ciprofloxacin. These Corynebacterineae isolates were used in the susceptibility study to broaden the information about drug resistance in this particular phylogenetic group, as the information available is still extremely limited. High MIC values were observed for three of the PANTA antimicrobials tested against Corynebacterium and Gordonia isolates. On the other hand, azlocillin and polymyxin B showed the lowest MICs (Table 2).

All tested aminoglycosides inhibited the in vitro growth of NTM and of the other Corynebacterineae, even at their lowest tested concentrations. Tobramycin, one of the two aminoglycosides considered in CLSI standards for NTM susceptibility testing [18] was an exception, with the rapidly growing $M$. obuense and $M$. mucogenicum isolates displaying resistant profiles (Table 2). High MIC values were obtained for spectinomycin, with all isolates being able to grow in the range of 4 to $64 \mu \mathrm{g} / \mathrm{mL}$ with the exception of one Gordonia isolate (1AIII) (MIC $<2 \mu \mathrm{g} / \mathrm{mL}$ ).

Although amphotericin B is an antifungal agent and no growth inhibition was expected for the bacterial isolates under study, the fact is that four of the NTM isolates were inhibited at the two highest amphotericin $B$ concentrations tested (Table 2). Divergent results between NTM and other Corynebacterineae were also observed regarding chloramphenicol and azlocillin with all NTM appearing to be resistant to these antibiotics, while MIC values for Corynebacterium and Gordonia isolates were in general much lower. Corynebacterium isolates were susceptible to vancomycin and Gordonia isolates presented similarly low MIC values. As expected with vancomycin MIC values for the NTM isolates were high, except for $M$. obuense that was surprisingly susceptible to this antibiotic and also to polymyxin B (Table 2). All of the NTM and Gordonia isolates were susceptible to linezolid. Corynebacterium isolates 6FIII and 58FIII were extremely resistant to penicillin (> 512 and $32 \mu \mathrm{g} / \mathrm{mL}$ ) (Table 2). 


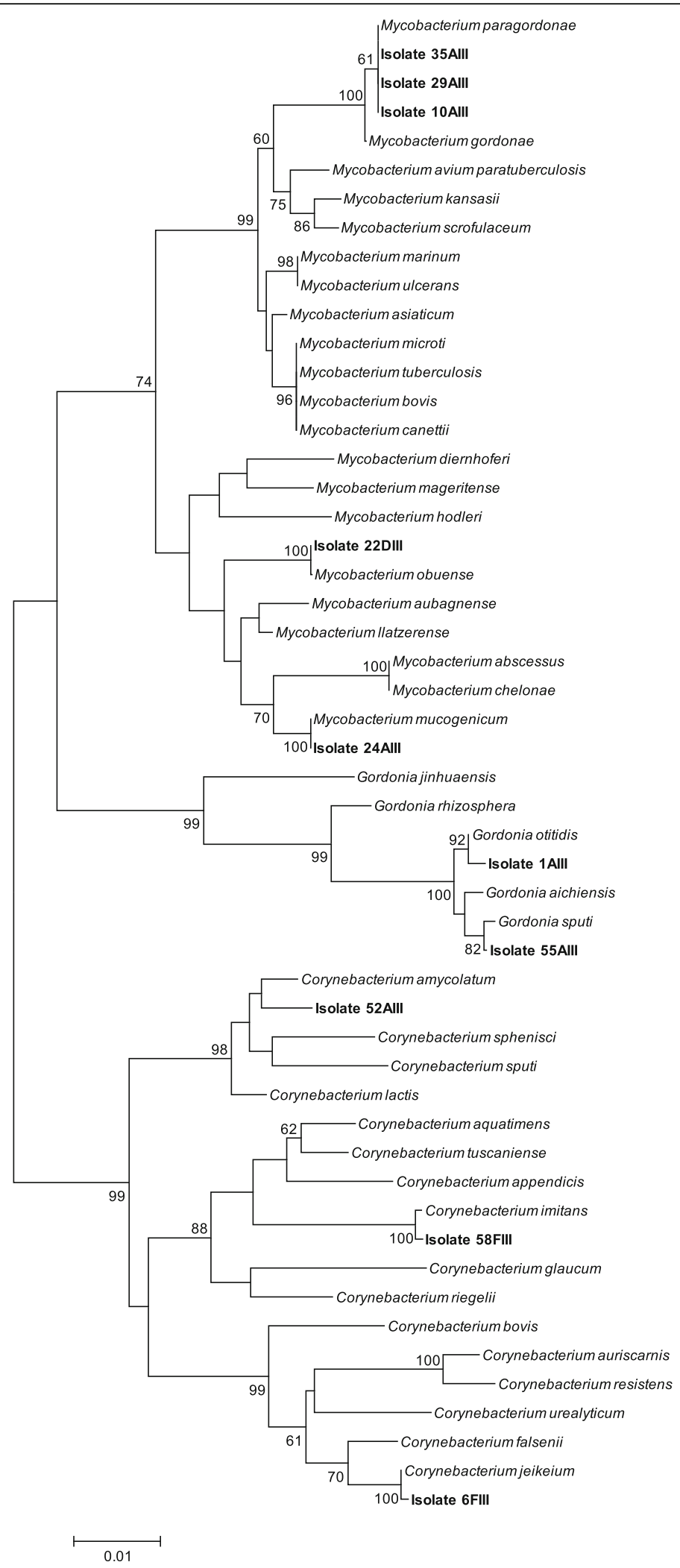

Fig. 1 (See legend on next page.) 
(See figure on previous page.)

Fig. 1 Phylogenetic tree based on a comparison of the $16 \mathrm{~S}$ rRNA gene sequences of isolates and their closest phylogenetic relatives belonging to the Corynebacterineae genera Mycobacterium, Corynebacterium and Gordonia (16S rRNA gene sequences of type strains available from GeneBank) (also see Additional file 1: Figure S1, Additional file 2: Figure S2 and Additional file 3: Figure S3). The tree was created using the neighbor-joining method. Bootstrap values above $60 \%$, for 500 replicates, are given at branch points. Bar, 1 inferred nucleotide substitutions per $100 \mathrm{nt}$

Results for ciprofloxacin, the only fluoroquinolone tested, ranged from high susceptibility in the 3 slowly growing NTM to the resistant phenotypes of the 2 rapidly growing NTM isolates (Table 2). Interestingly, high MIC values for ciprofloxacin were also obtained for the Corynebacterium isolates while the 2 Gordonia isolates had opposing results, one showed the lowest $(<0.125 \mu \mathrm{g} / \mathrm{mL})$ and the other had the highest $(16 \mu \mathrm{g} / \mathrm{mL}) \mathrm{MIC}$ values measured.

\section{Discussion}

Nosocomial infections are a major concern worldwide and represent an increase in hospital stay and treatment costs, particularly if associated with drug resistant pathogens [1]. The literature refers that probable dissemination vehicles between the pathogen niche (water faucets, medical instruments, fomites) and patients are mainly the healthcare providers [19].

Different wards of one hospital were sampled [17] and one third of the Actinobacterial isolates found belonged to the suborder Corynebacterineae, namely Corynebacterium amycolatum and C. jeikeium, Gordonia otitidis and G. sputi or Mycobacterium mucogenicum, all of which include potentially pathogenic strains previously implicated in human infections [20-22]. Of note was the fact that all samples were collected from sites with frequent human contact, placing these opportunistic pathogens easily accessible to patients, visitors and to healthcare providers. The surfaces of hospital amenities are considered important sources of pathogenic agents transmission [23] and the prevailing consensus indicates NTM opportunistic infections have environmental origin, although human-to-human transmission of $M$. abscessus is a factor of dissemination in cystic fibrosis patients [24, 25]. Isolation of M. mucogenicum has been mainly associated with hospital water distribution systems [26-31]. However, the isolate belonging to this species was recovered from a dry surface as were all the NTM strains studied here except the $M$. obuense isolate. Although $M$. avium strains are frequently isolated from waters, plumbing and showerheads biofilms, they are not, to the best of our knowledge, recovered frequently from surfaces such as those sampled in this study also possibly because their tolerance to desiccation appears to be low $[32,33]$.

The Mycobacterium strains recovered appeared to be relatively rare [17]. However, because their identification was initially based on 16S rRNA sequences alone, the putative M. gordonae isolates were now confirmed to be more closely related to the species $M$. paragordonae after concatenation of partial sequences of genes $16 \mathrm{~S}$ rRNA, $r p o B$ and $h s p 65$, which provided a stronger species association confirmed by the construction of the corresponding phylogenetic tree. The $M$. paragordonae species was originally described in 2014 based on a clinical isolate from a patient with a pulmonary infection [34]. Since then $M$. paragordonae was only isolated twice, both from healthcare settings $[35,36]$. The NTM species more frequently isolated from patients in Portugal in the last years were those in the M. avium complex, M. gordonae and M. kansasii [37]. Interestingly, our phylogenetic study shows that the three presumptive isolates initially identified as $M$. gordonae based on 16S rRNA sequencing [17], are in fact members of the recently described and rarely isolated species $M$. paragordonae, which raises questions about the true identity of clinical $M$. gordonae isolates, one of the species often recovered from patients in Portugal [37].

Mycobacterium obuense on the other hand appears to be common in soils and plants [38-40], but has been only rarely isolated from clinical samples [41-44] and, to our knowledge, there has been only one report of its isolation from a hospital environment [35]. Still, both species have been isolated from sputum of patients with pulmonary infections but, as is often the case for rarely isolated NTM [45], their clinical relevance remains uncertain. In addition to its clinical relevance and ability to cause a range of infections, $M$. mucogenicum has also been commonly implicated in nosocomial outbreaks [21, 46]. Indeed, its presence in the healthcare environment, if persistent, may pose a risk for patients. Although more prevalent than $M$. paragordonae and M. obuense, M. mucogenicum is still a rarely isolated species $[15,44]$.

The M. mucogenicum isolate showed a multidrug resistance (MDR) profile, at least to 4 different classes of antimicrobials (fluoroquinolones, tetracyclines, aminoglycosides and sulfonamides). This differs from what has been reported for the type strain $M$. mucogenicum DSM44625, which was found to be susceptible to ciprofloxacin, doxycycline and sulfamethoxazole [47], unlike the isolate in this study which was resistant to these 3 antibiotics. Furthermore, van Ingen and colleagues tested $15 \mathrm{M}$. mucogenicum clinical strains against a panel of 11 antibiotics and found the majority to be susceptible to rifabutin, amikacin, ciprofloxacin and clarithromycin [48]. The M. obuense isolate, also exhibited a MDR profile namely to ciprofloxacin, 


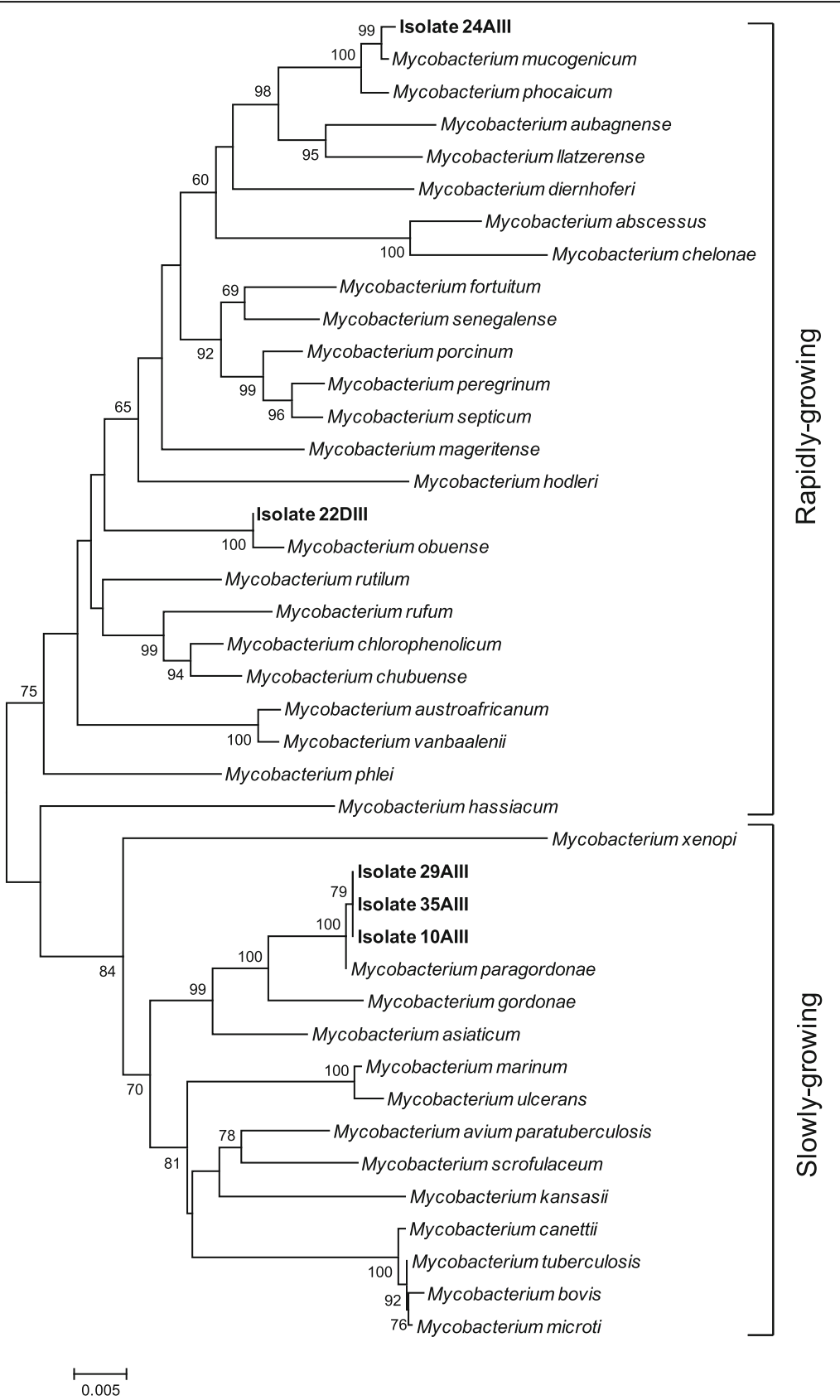

Fig. 2 Phylogenetic tree computed from the concatenated nucleotide sequences of $16 \mathrm{~S}$ rRNA, hsp65 and rpoB from Mycobacterium isolates and from strains selected from databases, using the neighbor-joining algorithm. The tree includes 41 strains after checking the congruence from each single-gene tree (see Additional file 1: Figure S1, Additional file 4: Figure S4 and Additional file 5: Figure S5). The evolutionary distances were calculated by the Jukes and Cantor method [61]. Bootstrap values above 60\%, for 500 replicates, are given at branch points. Bar, 5 inferred nucleotide substitutions per $1000 \mathrm{nt}$

clarithromycin, imipenem and to tobramycin, unlike the $M$. obuense type strain ATCC27023 [47] and unlike a clinical isolate [48] both susceptible to ciprofloxacin, cefoxitin, tobramycin and clarithromycin. In our study, the $M$. obuense isolate was the only NTM resistant to clarithromycin. We have sequenced the genomes of the 5 NTM recovered (unpublished results), and $M$. obuense was the only to possess a classical erm gene [49]. To our knowledge, no $M$. paragordonae strains have been tested for antibiotic susceptibility prior to this work and the isolates tested here were susceptible to 4 of the CLSI antibiotics recommended for slowly growing mycobacteria. Thus, 2 of the 5 NTM isolated in this study presented MDR profile, and were more drug resistant than the previously isolated strains of the same species. Multidrug-resistant NTM have been described in the literature but not originating from the hospital 
Table 2 MIC values $[\mu \mathrm{g} / \mathrm{mL}(\mathrm{U} / \mathrm{mL}$ for polymyxin B)] of 25 antimicrobials used for susceptibility testing of NTM and other Corynebacterineae

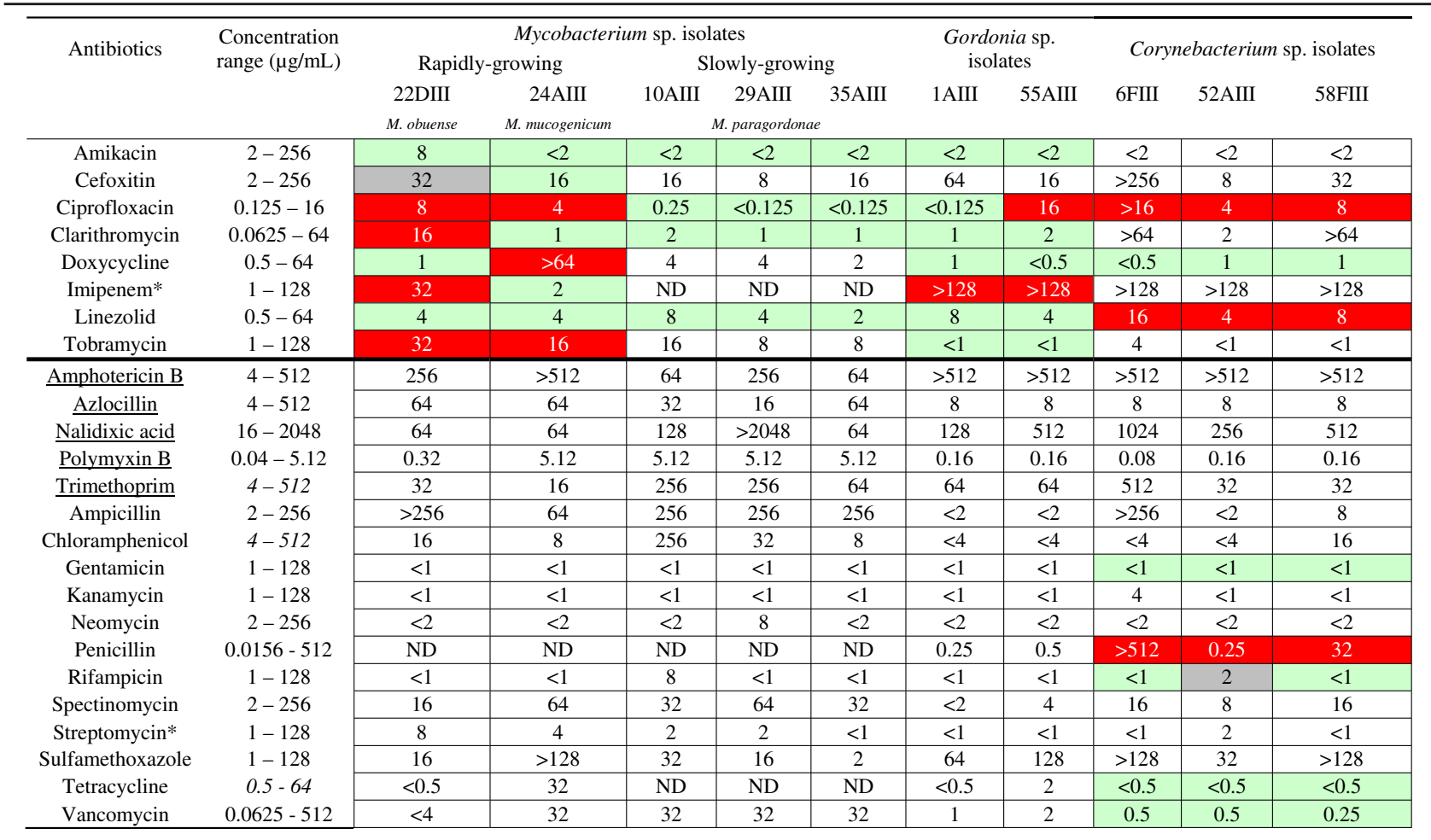

Resistant profile (red shading); intermediate profile (grey shading); susceptible profile (green shading), according to interpretative criteria [18, 68]. When no interpretive criteria are available only MIC values are reported. The dark line separates the antibiotics recommended by the CLSI to be tested for rapidly growing NTM (above the line) from the other 17 antimicrobials tested (below the line)

ND not determined

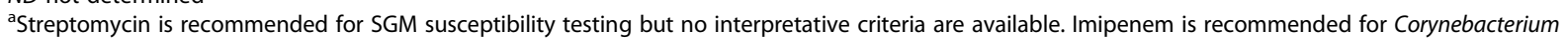
susceptibility testing but no interpretative criteria are available. The 5 antibiotics with underlined names were utilized in the Middlebrook 7H10-PANTA supplemented isolation medium

environment $[9,50]$. Intraspecific variability, infrequent isolation and lack of reports on drug resistance profiles all contribute to the difficulty in defining standard treatment guidelines for rare opportunistic NTM such as M. тисоgenicum [51]. Remarkably, all NTM isolates in this study were susceptible to the aminoglycosides tested, except for the 2 rapidly growing NTM isolates that were resistant to tobramycin.

Corynebacterium are in general resistant to antimicrobial agents recommended for Gram-positive infections, including penicillins, cephalosporins, macrolides, fluoroquinolones, aminoglycosides and tetracycline, but they remain susceptible to vancomycin and linezolid [52]. The 3 Corynebacterium isolates in this study showed an unusual resistance pattern, since they were susceptible to aminoglycosides and tetracyclines, but were also resistant to linezolid. Riegel et al. tested the susceptibility of 13 nosocomial $C$. jeikeium isolates against gentamicin, with half presenting a $\mathrm{MIC}<0.5 \mu \mathrm{g} / \mathrm{mL}$ and the other half a MIC $>16 \mu \mathrm{g} / \mathrm{mL}$ [53]. Scarce literature with low number of isolates hinders overall interpretation of results, which may be worth exploring for further awareness of antibiotic resistance in these increasingly detected potentially opportunistic pathogens.

One important observation from our study was the fact that all Corynebacterium and Gordonia isolates were highly resistant to imipenem. Although we did not assess the genetic background underlying this phenotype, it is possible to speculate that if the resistance is related to the presence of carbapenemases, these slowly growing bacteria can represent an unknown resistance pool against this important antibiotic. We found no literature reporting the presence of carbapenemases in species of the Corynebacterineae, but genetic mobile elements with different resistance genes have already been described in some of these species [54, 55], highlighting their ability to transfer antibiotic resistance features.

Only 5 of the 25 tested antimicrobials were those used in Middlebrook 7H10-PANTA culture medium for isolation although our results showed MICs much higher than the concentrations used in the selective medium. Possibly, increasing the concentration of these antimicrobials, 
except azlocillin, could diminish the number of false positives for the selection of NTM from the hospital environment, as the presence of a higher number of colony forming units per plate observed in some samples could have affected the recovery rate (data not shown). This is worth testing to optimize present standard methodology for NTM isolation with PANTA-supplemented medium [56]. Surprisingly, while Corynebacterium and Gordonia isolates were highly resistant to the antifungal amphotericin B, the MICs for most NTM isolates were intermediate confirming that at higher concentrations amphotericin B seems to inhibit their growth.

\section{Conclusions}

The current study, although limited in number of isolates, revealed the poor knowledge we still have on the identity of viable NTM species present in hospital settings, as well as on their antibiotic resistance profiles and resistance mechanisms, raising relevant questions about the potential threat these and other potential opportunistic pathogens may represent for example to immunocompromised inpatients. Their presence in dry surfaces with which healthcare providers, visitors and patients themselves contact frequently, accompanied by their apparent multidrug resistance profiles, should be further investigated to comprehensively understand this potentially latent menace and help prevent dissemination through implementation of better disinfection strategies and enforcement of enhanced policies.

\section{Materials and methods}

\section{Sample collection from hospital settings}

Samples were collected from different surfaces and equipment located at three different wards of a tertiary care hospital, as previously described [17]. Suspensions without pre-treatment were directly plated in solid Middlebrook 7H10-PANTA supplemented medium [Middlebrook 7H10 medium enriched with 10\% OADC (oleic acid, albumin, dextrose, catalase) and supplemented with polymyxin B $(40 \mathrm{U} / \mathrm{mL})$, amphotericin B $(4 \mu \mathrm{g} / \mathrm{mL})$, nalidixic acid $(16 \mu \mathrm{g} / \mathrm{mL})$, trimethoprim $(4 \mu \mathrm{g} / \mathrm{mL})$ and azlocillin $(4 \mu \mathrm{g} /$ $\mathrm{mL})][17,56]$. Plates were incubated at $30^{\circ} \mathrm{C}$ between 1 and 6 weeks and colony growth was evaluated on a weekly basis. Isolation, plating and purification of colonies was performed in Middlebrook 7H10-PANTA, followed by cryopreservation at $-80^{\circ} \mathrm{C}$ in Middlebrook $7 \mathrm{H} 9$ broth with $15 \%$ glycerol.

\section{Identification of NTM and other actinobacterial isolates}

Genomic DNA was extracted as previously described [57]. Amplification of the full-length 16S rRNA gene was performed by polymerase chain reaction (PCR) with universal primers 27F (5'-GAGTTTGATCCTGGCTCAG) and 1525R (5'-AGAAAGGAGGTGATCCAGCC) [58]. PCR reactions were carried out with Supreme NZYTaq DNA polymerase (NZYTech, Portugal) with 30 cycles of $1 \mathrm{~min}$ at $94^{\circ} \mathrm{C}, 1 \mathrm{~min}$ at $55^{\circ} \mathrm{C}$, and $1 \mathrm{~min}$ at $72^{\circ} \mathrm{C}$. Products were purified using JET Quick PCR Purification Spin Kit (Genomed GmbH, Germany) according to manufacturer's instructions and sequenced at GATC Biotech (Germany). $16 \mathrm{~S}$ rRNA gene sequences were compared with sequences at the NCBI database using the BLAST tool (http://blas t.ncbi.nlm.nih.gov/) and assignment to species level considered nucleotide sequence identities of $\geq 99 \%$. For species identity validation, DNA from Mycobacterium isolates was used for PCR amplification of partial sequences of $r p o B$ and $h s p 65$ genes with mycobacterial-specific primers GrpoB1 (5'-ATCGACCACTTCGGCAACCGCC), GrpoB2 (5'-GGTACGGCGTCTCGATGAASCCG), and Tb 11 (5'-ACCACGATGGTGTGTCCAT), Tb12 (5'-CTTG TCGAACCGCATACCCT), respectively [59]. PCR reactions were carried out with KOD Hot-Start DNA polymerase (Novagen) according to manufacturer's instructions and PCR products were purified and sequenced, as described above.

\section{Sequence analyses and phylogenetic trees}

Phylogenetic analyses were performed after manually checking DNA quality using Sequence Scanner Software (Applied Biosystems). Sequence data was edited and assembled with BioEdit Sequence Alignment Editor. The 16S rRNA gene sequences of the isolates and type strains of the Corynebacterineae genera Mycobacterium, Gordonia and Corynebacterium were obtained from Genbank or ARB Silva database (https://www.arb-sil va.de/) and aligned, each genus separately, with the Clustal X software package [60], visually examined and manually adjusted to allow maximal alignment. Jukes Cantor method was used to calculate evolutionary distances [61]. Phylogenetic dendrograms were constructed by the neighbor-joining method and evaluated by bootstrap analysis [62] of 500 resamplings of the data set, using MEGA6 software [63]. Three phylogenetic trees (Additional file 1: Figure S1, Additional file 2: Figure S2, Additional file 3: Figure S3, Additional file 4: Figure S4 and Additional file 5: Figure S5) were used to accurately determine the phylogenetic placement of the isolates for downstream selection of the Type strains to be used for the construction of the final tree (as described above) including the three genera belonging to Corynebacterineae (Fig. 1). The similarity values of the 16S rRNA gene sequences of the all isolates and the closest type strains were determined from the alignment used to construct the phylogenetic tree encompassing the three genera and are presented as Additional file 6: Table S1. The assignment to species level considered nucleotide sequence similarity value of $\geq 99 \%$ of the isolates towards the closest type strains. Amino acid sequences were deduced with the MEGA6 package from the 420- and 396-bp DNA 
sequences of mycobacterial partial $h s p 65$ and rpoB gene sequences, respectively. Protein sequences were aligned with sequences of type strains obtained from the NCBI database using the Clustal X. Protein phylogenetic trees were constructed using the neighbor-joining [64]. Topology of trees were generated from evolutionary distances computed using the Poisson correction method [65], included in Mega6 and evaluated by bootstrap analysis [62] of 500 resamplings of the data set. All positions with less than $95 \%$ site coverage were eliminated. Protein alignments were used to determine the nucleotide position in the DNA sequences alignment and sequences from mycobacterial genes $16 \mathrm{~S}$ rRNA, $h s p 65$ and $r p o B$ were concatenated and further used for phylogenetic analyses as described above.

To search for the clarithromycin resistance gene erm and for the tetracycline resistance gene tet $(V)$, chromosomal DNA from NTM isolates was used as template for draft genome sequencing at GATC Biotech (Konstanz, Germany) with $150 \mathrm{bp}$ paired-end libraries on an Illumina HiSeq. Raw sequence reads were assembled de novo using SPAdes 3.11.1 [66] with specific parameters for $2 \times 150 \mathrm{bp}$ reads library de novo assembly, namely using BayesHammer module error correction and --careful option (our unpublished results).

\section{Deposition of nucleic acid sequences in public databases} Partial 16S rRNA (1347-1378 bp) genes are available from [17] under accession numbers KT347497 and KT347499 to KT347502. Partial rpoB (371-398 bp) and hsp65 (395-441 bp) genes sequences were deposited in European Molecular Biology Laboratory (EMBL) and GenBank databases under the accession numbers: KT992215 to KT992224 for the partial rpoB and hsp65 sequences, respectively, and from KT832812 to KT832816 for the Corynebacterineae isolates partial 16S rRNA sequences. The clarithromycin resistance gene erm detected in the $M$. obuense genome and the tetracycline resistance genes $\operatorname{tet}(V)$ identified in $M$. obuense and $M$. mucogenicum draft genomes were deposited in GenBank database under accession numbers MG770427, MG770425 and MG770428, respectively.

\section{Antimicrobial susceptibility testing and minimal inhibitory concentration (MIC)}

Minimal inhibitory concentrations were determined after 5 days according to Clinical Laboratory Standards Institute (CLSI) recommendations for rapidly growing NTM and Nocardia [18, 67]. Corynebacterium isolates were incubated for $48 \mathrm{~h}$ according to CLSI recommendations [68]. The slowly growing M. paragordonae isolates 10AIII, 29AIII and 35AIII were incubated for 10 days. Clarithromycin susceptibility was determined after 14 days [69]. Classification of mycobacteria according to their growth rate is classically based on the time bacteria take to form colonies in solid media. Rapidly growing mycobacteria (RGM) are able to grow in under 7 days, whereas the ones that take more than 7 days are called slowly growing mycobacteria (SGM). Because phylogenetic studies of mycobacteria support this separation identification of SGM or RGM species was based on the phylogenetic tree constructed by Tortoli et al. [70] in addition to CLSI listing [18]. Briefly, a suspension of 0.5 McFarland density of each isolate was prepared in saline solution and diluted 1000 -fold before testing in the next $30 \mathrm{~min}$. A sterile 96-well microplate, previously prepared with Mueller Hinton $(\mathrm{MH})$ medium supplemented with $0.5 \%$ OADC and containing decreasing concentrations of the tested antimicrobials, was inoculated with the diluted bacterial suspension and incubated for 5 days at $30^{\circ} \mathrm{C}$ [18]. In addition to the antimicrobials considered for rapidly growing mycobacteria susceptibility testing in CLSI standards (cefoxitin, amikacin, imipenem, tobramycin, linezolid, doxycycline, clarithromycin and ciprofloxacin) also amphotericin B, azlocillin, nalidixic acid, trimethoprim, polymyxin B (these 5 used in Middlebrook 7H10-PANTA), rifampicin, chloramphenicol, tetracycline, penicillin, vancomycin and the aminoglycosides gentamicin, kanamycin, streptomycin, neomycin and spectinomycin were tested. Only antimicrobials considered in CLSI standards were interpreted for bacterial resistance levels [18, 68]. Diverse concentration ranges were used, with antimicrobials being diluted 8 times, in a 1:2 scaling (clarithromycin was diluted 12 times and penicillin was diluted 16 times). Stock solutions were prepared according CLSI guidelines [71, 72]. Appropriate controls were performed to ensure normal bacterial growth despite presence of diluted acetic acid, methanol or ethanol used to solubilize some antibiotics. All assays were performed in triplicate.

\section{Additional files}

Additional file 1: Figure S1. Phylogenetic dendrogram constructed by comparing $16 \mathrm{~S}$ rRNA gene sequences of isolates 10Alll, 22DIII, 24All, 29AIII and 35Alll with Mycobacterium type strain sequences obtained from GenBank databases. Sequences were aligned using MEGA6. The tree topology was obtained by using neighbor-joining algorithm with Jukes-Cantor correction. All positions with less than 95\% site coverage were eliminated. Bootstrap values above $60 \%$, for 500 replicates, are given at branch points. Bar, 1 inferred nucleotide substitution per $100 \mathrm{nt}$. (PPTX $108 \mathrm{~kb}$ )

Additional file 2: Figure S2. Phylogenetic dendrogram constructed by comparing 165 rRNA gene sequences of isolates 1 Alll and 55All with Gordonia type strain sequences obtained from databases. Sequences were aligned using MEGA6. The tree topology was obtained by using neighborjoining algorithm with Jukes-Cantor correction. All positions with less than $95 \%$ site coverage were eliminated. Bootstrap values above $60 \%$, for 500 replicates, are given at branch points. Bar, 5 inferred nucleotide substitution per $1000 \mathrm{nt}$. (PPTX $75 \mathrm{~kb}$ )

Additional file 3: Figure S3. Phylogenetic dendrogram constructed by comparing $16 \mathrm{~S}$ rRNA gene sequences of isolates 6FIII, 52Alll and 58FIII 
with Corynebacterium type strain sequences obtained from databases. Sequences were aligned using MEGA6. The tree topology was obtained by using neighbor-joining algorithm with Jukes-Cantor correction. All positions with less than $95 \%$ site coverage were eliminated. Bootstrap values above $60 \%$, for 500 replicates, are given at branch points. Bar, 1 inferred nucleotide substitution per $100 \mathrm{nt}$. (PPTX $95 \mathrm{~kb}$ )

Additional file 4: Figure S4. Phylogenetic analysis of $r p o B$ nucleotide sequences of mycobacterial isolates and 61 selected types strains of the genus Mycobacterium. The tree was created using the neighbor-joining algorithm and the evolutionary distances calculated by Jukes and Cantor method [61]. Bootstrap values above 60\%, for 500 replicates, are given at branch points. Bar, 1 inferred nucleotide substitution per $100 \mathrm{nt}$. (PPTX $92 \mathrm{~kb}$ )

Additional file 5: Figure S5. Phylogenetic analysis of hsp65 nucleotide sequences of mycobacterial isolates and the 55 selected type strains of the genus Mycobacterium. See Additional file 5: Figure S2 legend for further details. (PPTX $90 \mathrm{~kb}$ )

Additional file 6: Table S1. Pairwise similarity values (\%) determined from the alignment used for the construction of the phylogenetic trees from: A. The concatenated nucleotide sequences of mycobacterial $16 \mathrm{~S}$ rRNA, hsp 65 and rpoB of isolates and Type strains selected from the databases; B. 16S rRNA gene nucleotide sequences of isolates and Type strains of the genus Corynebacterium selected from the databases; $C$. 165 rRNA gene nucleotide sequences of isolates and Type strains of the genus Gordonia selected from the databases. (DOCX $48 \mathrm{~kb}$ )

\section{Abbreviations}

BLAST: Basic Local Alignment Search Tool; CFU: Colony forming units; CLSI: Clinical Laboratory Standards Institute; EMBL: European Molecular Biology Laboratory; HAl: Hospital-acquired infections; MDR: Multidrug resistance; MIC: Minimal inhibitory concentration; NCBI: National Center for Biotechnology Information; NTM: Nontuberculous mycobacteria; OADC: Oleic acid, albumin, dextrose, catalase; PANTA: polymyxin B $(40 \mathrm{U} / \mathrm{mL})$, amphotericin B $(4 \mu \mathrm{g} / \mathrm{mL})$, nalidixic acid $(16 \mu \mathrm{g} / \mathrm{mL})$, trimethoprim $(4 \mu \mathrm{g} / \mathrm{mL})$ and azlocillin; PCR: Polymerase chain reaction; WHO: World Health Organization

\section{Acknowledgements}

We thank Paula V. Morais (Life Sciences Department, University of Coimbra, Portugal), Ana M. Almeida (Instituto Piaget, Silves, Portugal) and the Infection Control office of the sampled tertiary hospital for their valuable collaboration.

\section{Funding}

We acknowledge Fundação para a Ciência e a Tecnologia (FCT) and COMPETE 2020, Operational Programme for Competitiveness and Internationalization (POCI) through FEDER projects UID/NEU/04539/2013 (POCI-01-0145-FEDER007440) and PTDC/BTM-TEC/29221/2017 (POCl-01-0145-FEDER-029221), and for postdoctoral fellowship SFRH/BPD/108299/2015 to Susana Alarico, for PhD fellowship SFRH/BD/117777/2016 to Daniela Nunes-Costa, and for FCT Investigator contract (IF/01061/2014) to Igor Tiago. We also acknowledge the European Regional Development Fund, Centro2020 Regional Operational Programme (CENTRO-01-0145-FEDER-000012-HealthyAging2020) for postdoctoral grant to Ana Maranha. The funding bodies listed here did not participate in the design of the study, collection, analysis, interpretation of data or in manuscript writing.

\section{Availability of data and materials}

All data generated or analyzed during this study are included in this article. All nucleic acid sequences were deposited in GenBank and the corresponding accession numbers can be found under "Deposition of nucleic acid sequences in public databases" in the Materials and Methods section.

\section{Authors' contributions}

SGP and AM performed MIC determination, data analysis and interpretation and were involved in manuscript writing. SA and IT constructed phylogenetic trees and were involved in manuscript writing. DR cryopreserved the isolates and participated in MIC determination experiments. DN-C performed DNA extraction and PCR and was involved in manuscript writing. OC interpreted data and was involved in manuscript writing. OC and NE contributed material and reagents. NE and AM designed the study, analyzed and interpreted data, and wrote the manuscript. All authors approved the final version of the manuscript.

\section{Ethics approval and consent to participate}

Not applicable.

\section{Consent for publication}

Not applicable.

\section{Competing interests}

The authors declare that they have no competing interests.

\section{Publisher's Note}

Springer Nature remains neutral with regard to jurisdictional claims in published maps and institutional affiliations.

\section{Author details}

${ }^{1}$ Center for Neuroscience and Cell Biology (CNC), University of Coimbra, 3004-504 Coimbra, Portugal. ${ }^{2}$ Institute for Interdisciplinary Research (IIIUC), University of Coimbra, 3030-789 Coimbra, Portugal. ${ }^{3}$ Centre for Functional Ecology (CFE), Department of Life Sciences, University of Coimbra, Coimbra, Portugal. ${ }^{4}$ PhD Program in Experimental Biology and Biomedicine (PDBEB), Institute for Interdisciplinary Research (IIIUC), University of Coimbra, 3030-789 Coimbra, Portugal. ${ }^{5}$ Faculty of Pharmacy, University of Coimbra, Coimbra, Portugal. ${ }^{6}$ Chemical Process Engineering and Forest Products Center (CIEPQPF), University of Coimbra, Coimbra, Portugal.

Received: 30 April 2018 Accepted: 26 February 2019

Published online: 19 March 2019

\section{References}

1. WHO. Report on the Burden of Endemic Health Care-Associated Infection Worldwide. Geneva: World Health Organization; 2011. http://apps.who.int/ iris/handle/10665/80135. Accessed 13 July 2017

2. Allegranzi B, Kilpatrick C, Storr J, Kelley E, Park BJ, Donaldson L, Global Infection P, Control N. Global infection prevention and control priorities 2018-22: a call for action. Lancet Glob Health. 2017;5(12):e1178-80.

3. ECDC. Healthcare-associated infections acquired in intensive care units annual epidemiological report 2016 [2014 data]. In: Annual Epidemiological Report on Communicable Diseases in Europe. Stockholm: European Centre for Disease Prevention and Control; 2017. https://ecdc.europa.eu/en/ publications-data/infections-acquired-intensive-care-units-annual-report2016. Accessed 13 July 2017.

4. Boucher HW, Talbot GH, Bradley JS, Edwards JE, Gilbert D, Rice LB, Scheld M, Spellberg B, Bartlett J. Bad Bugs, No Drugs: No ESKAPE! An update from the Infectious Diseases Society of America. Clin Infect Dis. 2009;48(1):1-12.

5. WHO. Global priority list of antibiotic-resistant bacteria to guide research, discovery, and development of new antibiotics. Geneva: World Health Organization; 2017. http://www.who.int/medicines/publications/globalpriority-list-antibiotic-resistant-bacteria/en/

6. Fedrizzi T, Meehan CJ, Grottola A, Giacobazzi E, Fregni Serpini G, Tagliazucchi S, Fabio A, Bettua C, Bertorelli R, De Sanctis V, et al. Genomic characterization of nontuberculous mycobacteria. Sci Rep. 2017;7:45258

7. Falkinham JO III. Current epidemiologic trends of the nontuberculous mycobacteria (NTM). Curr Environ Health Rep. 2016;3(2):161-7.

8. Griffith DE, Aksamit T, Brown-Elliott BA, Catanzaro A, Daley C, Gordin F, Holland SM, Horsburgh R, Huitt G, lademarco MF, et al. An official ATS/IDSA statement: diagnosis, treatment, and prevention of nontuberculous mycobacterial diseases. Am J Respir Crit Care Med. 2007;175(4):367-416.

9. Brown-Elliott BA, Nash KA, Wallace RJ. Antimicrobial susceptibility testing, drug resistance mechanisms, and therapy of infections with nontuberculous mycobacteria. Clin Microbiol Rev. 2012:25(3):545-82.

10. Tuttle MS, Mostow E, Mukherjee P, Hu FZ, Melton-Kreft R, Ehrlich GD, Dowd SE, Ghannoum MA. Characterization of bacterial communities in venous insufficiency wounds by use of conventional culture and molecular diagnostic methods. J Clin Microbiol. 2011;49(11):3812-9.

11. Russotto V, Cortegiani A, Raineri SM, Giarratano A. Bacterial contamination of inanimate surfaces and equipment in the intensive care unit. J Intensive Care. 2015:3:54

12. LPSN-list of prokaryotic names with standing in nomenclature. 1997 www.bacterio.net/mycobacterium.html. Accessed 10 Dec 2018

13. lida S, Taniguchi H, Kageyama A, Yazawa K, Chibana H, Murata S, Nomura F, Kroppenstedt RM, Mikami Y. Gordonia otitidis sp. nov., isolated from a patient with external otitis. Int J Syst Evol Microbiol. 2005;55(5):1871-6. 
14. Biswal I, Mohapatra S, Deb M, Dawar R, Gaind R. Corynebacterium striatum: an emerging nosocomial pathogen in a case of laryngeal carcinoma. Indian J Med Microbiol. 2014;32(3):323-4.

15. van der Werf M, Kodmon C, Katalinic-Jankovic V, Kummik T, Soini H, Richter E, Papaventsis D, Tortoli E, Perrin M, van Soolingen D, et al. Inventory study of nontuberculous mycobacteria in the European Union. BMC Infect Dis. 2014;14(1):62

16. Nunes-Costa D, Alarico S, Dalcolmo MP, Correia-Neves M, Empadinhas N. The looming tide of nontuberculous mycobacterial infections in Portugal and Brazil. Tuberculosis. 2016;96:107-19.

17. Geadas Farias P, Gama F, Reis D, Alarico S, Empadinhas N, Martins JC, de Almeida AF, Morais PV. Hospital microbial surface colonization revealed during monitoring of Klebsiella spp., Pseudomonas aeruginosa, and non-tuberculous mycobacteria. Antonie Van Leeuwenhoek. 2017; 110(7):863-76.

18. CLSI. Susceptibility testing of Mycobacteria, Nocardia, and other aerobic Actinomycetes; approved standard. In., vol. document M24-A, 2nd edn. Wayne: Clinical and Laboratory Standards Institute; 2011.

19. Cummings KL, Anderson DJ, Kaye KS. Hand hygiene noncompliance and the cost of hospital-acquired methicillin-resistant Staphylococcus aureus infection. Infect Control Hosp Epidemiol. 2010;31(4):357-64.

20. Lai CC, Wang CY, Liu CY, Tan CK, Lin SH, Liao CH, Chou CH, Huang YT, Lin $\mathrm{HI}$, Hsueh PR. Infections caused by Gordonia species at a medical Centre in Taiwan, 1997 to 2008. Clin Microbiol Infect. 2010;16(9):1448-53.

21. Adekambi T. Mycobacterium mucogenicum group infections: a review. Clin Microbiol Infect. 2009;15(10):911-8.

22. Belmares J, Detterline S, Pak JB, Parada JP. Corynebacterium endocarditis species-specific risk factors and outcomes. BMC Infect Dis. 2007;7:4-4.

23. Weber DJ, Rutala WA, Miller MB, Huslage K, Sickbert-Bennett E. Role of hospital surfaces in the transmission of emerging health care-associated pathogens: norovirus, Clostridium difficile, and Acinetobacter species. Am J Infect Control. 2010;38(5 Suppl 1):S25-33.

24. Bryant JM, Grogono DM, Rodriguez-Rincon D, Everall I, Brown KP, Moreno P, Verma D, Hill E, Drijkoningen J, Gilligan P, et al. Emergence and spread of a human-transmissible multidrug-resistant nontuberculous mycobacterium. Science. 2016;354(6313):751-7.

25. Walker TM, Crook DW, Peto TEA, Conlon CP. Whole-genome sequencing identifies nosocomial transmission of extra-pulmonary M. tuberculosis. QJM. 2016;109(12):819-20

26. Crago B, Ferrato C, Drews SJ, Louie T, Ceri H, Turner RJ, Roles A, Louie M. Surveillance and molecular characterization of non-tuberculous mycobacteria in a hospital water distribution system over a three-year period. J Hosp Infect. 2014;87(1):59-62.

27. Genc GE, Richter E, Erturan Z. Isolation of nontuberculous mycobacteria from hospital waters in Turkey. APMIS. 2013;121(12):1192-7.

28. Sartori FG, Leandro LF, Montanari LB, de Souza MGM, Pires RH, Sato DN, Leite CQF, de Andrade PK, Martins CHG. Isolation and identification of environmental mycobacteria in the waters of a hemodialysis center. Curr Microbiol. 2013;67(1):107-11.

29. Fernandez-Rendon E, Cerna-Cortes JF, Ramirez-Medina MA, Helguera-Repetto AC, Rivera-Gutierrez S, Estrada-Garcia T, Gonzalez-y-Merchand JA. Mycobacterium mucogenicum and other non-tuberculous mycobacteria in potable water of a trauma hospital: a potential source for human infection. J Hosp Infect. 2012;80(1):74-6.

30. Souza MG, Sato DN, Leite CQF, Leite SR, Sartori FG, Prince K, Casmeiro LA, Martins $\mathrm{CH}$. Occurrence of pathogenic environmental mycobacteria on surfaces in health institutions. Res Rep Trop Med. 2010;2010(1):53-8.

31. Shin JH, Lee EJ, Lee HR, Ryu SM, Kim HR, Chang CL, Kim YJ, Lee JN. Prevalence of non-tuberculous mycobacteria in a hospital environment. J Hosp Infect. 2007;65(2):143-8.

32. Falkinham JO III, Pruden A, Edwards M. Opportunistic premise plumbing pathogens: increasingly important pathogens in drinking water. Pathogens. 2015:4(2):373-86

33. Archuleta $R$, Mullens $P$, Primm TP. The relationship of temperature to desiccation and starvation tolerance of the Mycobacterium avium complex. Arch Microbiol. 2002;178(4):311-4.

34. Kim BJ, Hong SH, Kook YH, Kim BJ. Mycobacterium paragordonae sp. nov., a slowly growing, scotochromogenic species closely related to Mycobacterium gordonae. Int J Syst Evol Microbiol. 2014;64(Pt 1):39-45.

35. Azadi D, Shojaei H, Pourchangiz M, Dibaj R, Davarpanah M, Naser AD. Species diversity and molecular characterization of nontuberculous mycobacteria in hospital water system of a developing country. Iran Microb Pathog. 2016;100:62-9.

36. Schreiber PW, Kuster SP, Hasse B, Bayard C, Rüegg C, Kohler P, Keller PM, Bloemberg GV, Maisano F, Bettex D, et al. Reemergence of mycobacterium chimaera in heater-cooler units despite intensified cleaning and disinfection protocol. Emerg Infect Dis. 2016;22(10):1830-3.

37. Ruas RR, Abreu I, Nuak J, Ramos A, Carvalho T, Ribeiro M, Guimaraes JT, Sarmento A. Nontuberculous mycobacteria in a tertiary Hospital in Portugal: a clinical review. Int J Mycobacteriol. 2017;6(4):344-8.

38. Koskimäki JJ, Hankala E, Suorsa M, Nylund S, Pirttilä AM. Mycobacteria are hidden endophytes in the shoots of rock plant [Pogonatherum paniceum (Lam.) Hack.] (Poaceae). Environ Microbiol Rep. 2010;2(4):619-24.

39. Laukkanen $H$, Soini $H$, Kontunen-Soppela S, Hohtola A, Viljanen M. A mycobacterium isolated from tissue cultures of mature Pinus sylvestris interferes with growth of scots pine seedlings. Tree Physiol. 2000; 20(13):915-20.

40. Tsukamura M, Mizuno S. Mycobacterium obuense, a rapidly growing scotochromogenic mycobacterium capable of forming a black product from p-aminosalicylate and salicylate. J Gen Microbiol. 1971;68(2):129-34.

41. Ford ES, Horne DJ, Shah JA, Wallis CK, Fang FC, Hawn TR. Species-specific risk factors, treatment decisions, and clinical outcomes for laboratory isolates of less common nontuberculous mycobacteria in Washington state. Ann Am Thorac Soc. 2017;14(7):1129-38.

42. Greninger AL, Cunningham G, Hsu ED, Yu JM, Chiu CY, Miller S. Draft genome sequence of Mycobacterium obuense strain UC1, isolated from patient sputum. Genome Announc. 2015:3(3):e00612-5.

43. Buijtels PCAM, Iseman MD, Parkinson $S$, de Graaff CS, Verbrugh HA, Petit PLC, van Soolingen D. Misdiagnosis of tuberculosis and the clinical relevance of non-tuberculous mycobacteria in Zambia. Asian Pac J Trop Med. 2010;3(5):386-91.

44. Martin-Casabona N, Bahrmand AR, Bennedsen J, Thomsen VO, Curcio M, Fauville-Dufaux M, Feldman K, Havelkova M, Katila ML, Koksalan K, et al. Non-tuberculous mycobacteria: patterns of isolation. A multi-country retrospective survey. Int J Tuberc Lung Dis. 2004:8(10):1186-93.

45. Kim J, Seong M-W, Kim E-C, Han SK, Yim J-J. Frequency and clinical implications of the isolation of rare nontuberculous mycobacteria. BMC Infect Dis. 2015;15(1):9.

46. Brown-Elliott BA, Philley JV. Rapidly Growing Mycobacteria. Microbiol Spectr. 2017;5(1). http://www.asmscience.org/content/journal/ microbiolspec/10.1128/microbiolspec.TNMI7-0027-2016.

47. Li G, Pang H, Guo Q, Huang M, Tan Y, Li C, Wei J, Xia Y, Jiang Y, Zhao X, et al. Antimicrobial susceptibility and MIC distribution of 41 drugs against clinical isolates from China and reference strains of nontuberculous mycobacteria. Int J Antimicrob Agents. 2017;49(3):364-74.

48. van Ingen J, van der Laan T, Dekhuijzen $R$, Boeree $M$, van Soolingen D. In vitro drug susceptibility of 2275 clinical non-tuberculous Mycobacterium isolates of 49 species in the Netherlands. Int J Antimicrob Agents. 2010;35(2):169-73.

49. Soetaert K, Rens C, Wang X-M, De Bruyn J, Lanéelle M-A, Laval F, Lemassu A, Daffé $M$, Bifani $P$, Fontaine $V$, et al. Increased vancomycin susceptibility in mycobacteria: a new approach to identify synergistic activity against multidrug-resistant mycobacteria. Antimicrob Agents Chemother. 2015;59(8):5057-60.

50. Candido PH, Nunes LS, Marques EA, Folescu TW, Coelho FS, de Moura VC, da Silva MG, Gomes KM, Lourenco MC, Aguiar FS, et al. Multidrug-resistant nontuberculous mycobacteria isolated from cystic fibrosis patients. J Clin Microbiol. 2014;52(8):2990-7.

51. Brown-Elliott BA, Wallace RJ. Clinical and taxonomic status of pathogenic nonpigmented or late-pigmenting rapidly growing mycobacteria. Clin Microbiol Rev. 2002;15(4):716-46.

52. Humphries RM, Hindler JA. Susceptibility test methods: fastidious bacteria. In: Jorgensen JH, Pfaller MA, Carroll KC, Funke G, Landry ML, Richter SS, Warnock DW, editors. Manual of clinical microbiology. 11th ed. Washington, DC: American Society of Microbiology; 2015. p. 1314-41.

53. Riegel $P$, de Briel D, Prévost $G$, Jehl F, Monteil H. Genomic diversity among Corynebacterium jeikeium strains and comparison with biochemical characteristics and antimicrobial susceptibilities. J Clin Microbiol. 1994;32(8):1860-5.

54. Tauch A, Krieft S, Pühler A, Kalinowski J. The tetAB genes of the Corynebacterium striatum R-plasmid pTP10 encode an ABC transporter and confer tetracycline, oxytetracycline and oxacillin resistance in Corynebacterium glutamicum. FEMS Microbiol Lett. 1999;173(1):203-9. 
55. Tauch A, Krieft S, Kalinowski J, Puhler A. The 51,409-bp R-plasmid pTP10 from the multiresistant clinical isolate Corynebacterium striatum M82B is composed of DNA segments initially identified in soil bacteria and in plant, animal, and human pathogens. Mol Gen Genet. 2000;263(1):1-11.

56. Radomski N, Cambau E, Moulin L, Haenn S, Moilleron R, Lucas FS. Comparison of culture methods for isolation of nontuberculous mycobacteria from surface waters. Appl Environ Microbiol. 2010;76(11):3514-20.

57. Alarico S, Costa M, Sousa MS, Maranha A, Lourenco EC, Faria TQ, Ventura MR, Empadinhas N. Mycobacterium hassiacum recovers from nitrogen starvation with up-regulation of a novel glucosylglycerate hydrolase and depletion of the accumulated glucosylglycerate. Sci Rep. 2014;4:6766.

58. Rainey FA, Ward-Rainey N, Kroppenstedt RM, Stackebrandt E. The genus Nocardiopsis represents a phylogenetically coherent taxon and a distinct actinomycete lineage: proposal of Nocardiopsaceae fam. nov. Int J Syst Bacteriol. 1996:46(4):1088-92.

59. Devulder G, de Montclos MP, Flandrois JP. A multigene approach to phylogenetic analysis using the genus Mycobacterium as a model. Int J Syst Evol Microbiol. 2005;55(1):293-302.

60. Larkin MA, Blackshields G, Brown NP, Chenna R, McGettigan PA, McWilliam H, Valentin F, Wallace IM, Wilm A, Lopez R, et al. Clustal W and Clustal X version 2.0. Bioinformatics. 2007;23(21):2947-8.

61. Jukes TH, Cantor CR. Evolution of protein molecules. In: Munro HN, editor. Mammalian protein metabolism. New York: Academic Press; 1969. p. 21-132.

62. Felsenstein J. Confidence limits on phylogenies: an approach using the bootstrap. Evolution. 1985;39(4):783-91.

63. Tamura K, Stecher G, Peterson D, Filipski A, Kumar S. MEGA6: molecular evolutionary genetics analysis version 6.0. Mol Biol Evol. 2013;30(12):2725-9.

64. Saitou N, Nei M. The neighbor-joining method: a new method for reconstructing phylogenetic trees. Mol Biol Evol. 1987;4(4):406-25.

65. Zuckerkandl E, Pauling L. Evolutionary divergence and convergence in proteins. In: Bryson V, Vogel HJ, editors. Evolving genes and proteins. New York: Academic Press; 1965. p. 97-166.

66. Bankevich A, Nurk S, Antipov D, Gurevich AA, Dvorkin M, Kulikov AS, Lesin VM, Nikolenko SI, Pham S, Prjibelski AD, et al. SPAdes: a new genome assembly algorithm and its applications to single-cell sequencing. J Comput Biol. 2012;19(5):455-77.

67. Woods GL, Lin S-YG, Desmond EP. Susceptibility test methods: mycobacteria, nocardia, and other actinomycetes. In: Jorgensen JH, Pfaller MA, Carroll KC, Funke G, Landry ML, Richter SS, Warnock DW, editors. Manual of clinical microbiology. 11th ed. Washington, DC: American Society of Microbiology; 2015. p. 1356-78.

68. CLSI. Methods for antimicrobial dilution and disk susceptibility testing of infrequently isolated or fastidious bacteria. In., 3rd edn. Wayne: Clinical and Laboratory Standards Institute; 2015.

69. Brown-Elliott BA, Vasireddy S, Vasireddy R, lakhiaeva E, Howard ST, Nash K, Parodi N, Strong A, Gee M, Smith T, et al. Utility of sequencing the erm (41) gene in isolates of Mycobacterium abscessus subsp. abscessus with low and intermediate clarithromycin MICs. J Clin Microbiol. 2015;53(4):1211-5.

70. Tortoli E, Fedrizzi T, Meehan CJ, Trovato A, Grottola A, Giacobazzi E, Serpini GF, Tagliazucchi S, Fabio A, Bettua C, et al. The new phylogeny of the genus Mycobacterium: the old and the news. Infect Genet Evol. 2017;56:19-25.

71. Barry A, Bryskier A, Traczewski M, Brown S. Preparation of stock solutions of macrolide and ketolide compounds for antimicrobial susceptibility tests. Clin Microbiol Infect. 2004;10(1):78-83.

72. CLSI. Performance standards for antimicrobial susceptibility testing, vol. M100. 27th ed. Wayne: Clinical and Laboratory Standards Institute; 2017.

Ready to submit your research? Choose BMC and benefit from:

- fast, convenient online submission

- thorough peer review by experienced researchers in your field

- rapid publication on acceptance

- support for research data, including large and complex data types

- gold Open Access which fosters wider collaboration and increased citations

- maximum visibility for your research: over $100 \mathrm{M}$ website views per year

At BMC, research is always in progress.

Learn more biomedcentral.com/submissions 